\title{
ENSAYOS
}




\section{EL ANALISIS DE CONVERSACION Y LA EDUCACION PRIMARIA}

D.R. Watson Ph.D*

\section{CONSIDERACIONES PRELIMINARES}

En este artículo se desea esbozar una perspectiva sociológica, metodológicamente radical de reciente desarrollo Se descubrirán su orígenes, sus presupuestos, su aplicación en la esfera de la educación primaria y se indicarán así mismo algunos de sus potenciales pertenencias para la exploración futura en este campo. Aunque se hacen referencias a algunos sistemas educativos específicos, como todo artículo que presente una perspectiva sociológica general, su validez desborda dichos casos.

\section{ETNOMETODOLOGIA Y ANALISIS DE CONVERSACION}

El análisis de conversación (conocido también como análisis conversacionall) ${ }^{* *}$ fue propuesto por Harvey Sacks y sus colegas de la Universidad de California en los Angeles (UCLA) durante la década del setenta. Con excepción de Roy Turner, quien pasó a trabajar al departamento de sociología de la Universidad de Columbia Británica en Vancouver en donde permanece hasta la fecha, los otros pioneros de este campo continúan en California. Sacks, estaba asociado con Harold Garfinkel también de UCLA, el menor de la perspectiva sociológica conocida como etnometodología.

La etnometodología corresponde a un rompimiento definitivo con las sociologías neokantianas que buscaban una descripción literal de los fenómenos sociales (por ejemplo, los fenómenos que incluyen la educación como una institución social), concebidos como si existieran de manera objetiva, es decir, independientes de las percepciones que cl observador -analista tiene de esos fenómenos. Garfinkel notó que un aspecto intrínseco de esas sociologías neo-kantianas era que privilegiaban las definiciones que el analista tenía de esos fenómenos, dado que eran aceptadas como "científicas", "literales" y "objetivas", mientras que las definiciones de los miembros de la sociedad, fundadas en el sentido común eran consistentemente consideradas como inadecuadas, incompletas y sesgadas. Estas definiciones, son consecuentemente desagregadas y descartadas tal como es el caso de la noción marxista de falsa conciencia, tan frecuentemente invocada en la sociología marxista de la educación, o la noción funcionalista de función / desfunción latente, las cuales son supuestamente desconocidas por los miembros de base de una sociedad. Mediante la adaptación de la filosofía del fenómeno del fenomenólogo Alfred Schutz. Garfinkel buscó trazar una aproximación empírica -sociológica que permitiera evitar esta "instancia irónica" del analista, esta actitud de competencia con las concepciones básicas que lo llevaban a considerar a los miembros ordinarios de una sociedad como dopados culturales.

La aproximación no-irónica y no competitiva de Garfinkel (ver Garfinkel 1984, Heriage 1984, Cuff y Payne 1985; Capítulo V), involucraba una revisión radical de los modelos

\footnotetext{
* El autor es Senior Lecturer del Departamento de Sociología de la Universidad de Manchester.

NT En Inglés Conversation (Conversational) Analysis.
} 
sociológicos sobre los miembros de una sociedad. Los miembros eran vistos como personas que poseían y compartían un cuerpo complejo y elaborado de conocimiento cultural -práctico, un conocimiento en elaboración (working Knowledge) que comprende "lo que todo el mundo sabe" en el cual los miembros están moralmente obligados a aceptar, usar y exhibir en el contexto de la sociedad. A diferencia de los interaccionistas simbólicos, Gafinkel afirma que este conocimiento era por naturaleza, de procedimiento más que sustantivo: conocer el cómo, más que exclusivamente conocer el qué.

Consecuentemente, Garfinkel se enfocaba al cómo de los problemas, por ejemplo, cómo los miembros construyen su conducta ordinaria, cómo articulan su conducta con la de los otros. Cómo crean un ordenamiento reconocible y familiar en sus contextos cotidianos. En resumen, él deseaba examinar los métodos culturalmente definidos (por ejemplo procedimientos de razonamiento cultural o prácticas para hacer sentido), mediante los cuales los miembros corrientes captaban, como asuntos reconocibles, las relaciones organizadas de las acciones sociales.

La etnometodología se refiere por lo tanto a un objeto de estudio (etno-gente y metodología -métodos o procedimientos), más que al delineamiento de un programa analítico per se: aunque es obvio que el primero determina de manera significativa los términos del segundo.

Sacks tomó y adaptó la aproximación de Garfinkel y su compromiso metodológico con la aproximación no irónica de este último y la expresa de manera muy adecuada cuando afirmaba:

"uno encuentra que todos (los pensadores revolucionarios desde la época presocrática hasta Freud) comenzaron con una expresión como la siguiente: Acerca de las cosas sobre las que voy a hablar, la gente piensa que sabe, pero ellos no saben. Más aún, si usted se los dice no cambian nada; siguen dando vueltas como si supieran, a pesar de que continúan caminando en un mundo soñado. Darwin empezó de esta forma, Freud lo hizo de manera similar." (paréntesis del autor)

En contraste con las aproximaciones de Freud y sus seguidores para analizar el conocimiento fundado en el sentido común que se construye en acción social (incluyendo especialmente el de tipo comunicativo), Sacks afirma: "en lo que estamos interesados es en lo que la gente conoce y usa" citado por Heritage, 1984: 23.

En particular, Sacks se preguntaba como los sociólogos podían obtener información de las acciones que ocurren, se organizan y se interrelacionan de una manera espontánea* que fuera susceptible de una inspección analítica, exhaustiva y repetible de manera tal que pudiera sustentar formulaciones sistemáticamente demostrables, chequeables y duplicables. Su trabajo fue sociológico en términos genéricos más que fundado en la psicología social o en la lingüística (ver la introducción de Lee a su libro editado con But!on, 1987). En un artículo de 1963, Sacks intentaba mostrar que los sociólogos no podían proceder de manera adecuada con su trabajo sin una caracterización analítica exhaustiva del aparato cultural (conocimiento en proceso) de los miembros de la sociedad, cuyos eventos se describían, tal como por ejemplo el evento suicidio. Dado que términos como el suicidio son términos lingüísticos para unos determinados cursos de acción, Sacks plantea la consideración de trabajar de manera extensiva con el lenguaje, como un fenómeno de interacción social.

\footnotetext{
${ }^{*}$ NT En el sentido de la actividad corriente y cotidiana de los miembros de una sociedad.
} 


\section{EL LENGUAJE Y LOS METODOS CULTURALES DE LOS MIEMBROS DE UNA SOCIEDAD}

Desde sus primeros trabajos Garfinkel insistía en que los sociólogos no pueden estudiar los aspectos pertinentes al razonamiento cultural del sentido común (el razonamiento práctico), sin examinar la destreza que alcanzan con el lenguaje "natural" los miembros de una sociedad en el proceso de producir actividades reconocibles.

El orden social (por ejemplo el Ordenamiento de las instituciones educativas), es por encima de todas las cosas un orden de tipo comunicativo. Algunas consideraciones sobre la manera como esta posición afecta la preocupación del analista con el lenguaje en el contexto educativo, puede consultarse en Heap, 1985.

Sacks buscaba también describir de manera rigurosa y empírica los componentes dc esta habilidad y demostrar que el conocimiento lingüístico de los miembros de una sociedad, era un componente integral de su capacidad para interactuar (ver por ejemplo la introducción de Lee ya citada).

La conversación ordinaria por ejemplo, opera a través de un conjunto de artificios interaccionales, los cuales fueron denominados por Sacks como el sistema de turnos, o de intercambio de discursos. El manejo interaccional de este sistema (y de sus sistemas derivados) por parte de los interlocutores, es el objeto del análisis conversacional (Sacks, Schegloff y Jeffersson,1974).

El término análisis de conversación hace referencia a un tópico de investigación analítica, es decir al propio análisis en curso que hacen los interlocutores de sus acciones de conversación.

Un aspecto central del abordaje de Sacks, es su rechazo de todas las formas de idealización, suposición, tipología ideal o datos imaginados, construidos exclusivamente por medio de una intuición nativa, que conlleven el reemplazo del mundo real adecuadamente captado de manera tal (datos filmados o grabados cuidadosamente transcritos), que preserven el trabajo lingüístico de los interlocutores como una interacción social por "naturaleza" (ver la introducción de los editores al primer artículo de Sacks publicado en Atkinson y Heritage, 1984).

A medida que se incrementaba el volumen de análisis de conversación, también crecía el interés por los sistemas de intercambio de discursos que se derivan del análisis anterior, y que de manera significativa transformaban la conversación cotidiana. En particular comenzaron a proliferar los estudios de los salones de clase en las escuelas y otros contextos de la educación formal Este creciente interés en la interacción comunicativa de los espacios educacionales no se limitaba solamente a los sistemas de intercambio de discursos, sino que también se preocupaba por la interacción niño -adulto y la naturaleza de la socialización infantil, aspectos que de muchas formas preservaban algunos de los supuestos genéricos de la etnometodología al poner en entredicho presupuestos sobre el aletargamiento cultural e insensatez inherentes a la comunicación entre los miembros de una sociedad.

\section{NIÑOS, ADULTOS Y SOCIALIZACION.}

En las sociologías ortodoxas y especialmente en el funcionalismo normativo, la noción de socialización presupone un modelo especial de niñez y madurez. Corno lo ha mostrado 
Robert Mackay de la Universidad de Toronto, el niño es considerado como una tabula rasa o una cavidad vacia que debe llenarse progresivamente con normas y valores en el proceso de socialización.

Dado que en estas sociologías convencionales los niños son concebidos esencialmente como sistemas deficitarios, los adultos son entonces vistos como los socializadores de los niños y por lo tanto, el proceso de socialización se visualiza en una sola dirección a saber: de los adultos hacia los niños. Consecuentemente, si las actividades y las concepciones de los niños son de alguna manera examinadas, tienden a ser definidas por los sociólogos como copias pobres de las actividades y concepciones de los adultos (casa de juguete, almacén de juguete, etc.). Las expresiones lingüísticas de los niños, concebidos como culturalmente inmaduros, tienden a ser concebidos por las sociologías ortodoxas, como réplicas incompletas o con errores de las expresiones adultas competentemente producidas.

Mackay señala algunas limitaciones en las concepciones que los sociólogos convencionales tienen de la socialización de los niños, definido (como si sólo comprendiera la internalización por parte de éstos de las normas provistas por los adultos, de manera tal que ellos/as crecen para ser esencialmente un facsímile del agente socializador adulto. Esta concepción presupone en primer lugar el carácter de la interacción adulto-niño y deja de lado una visión más compleja de la misma, particularmente en la medida en que no propicie el que los analistas inspeccionen en detalle las interacciones que de hecho ocurren; la concepción convencional promulgada por analistas como Talcott Parsons, comprende una caracterización analíticamente imaginaria o teóricamente dada, que no se basa en procesos observados. La aproximación de los sociólogos convencionales a la socialización, potencia por lo tanto una estrategia que ignora y que no se basa en la observación.

Así mismo, Mackay señala que el modelo ortodoxo de socialización define al niño como un recipiente pasivo y un imitador de las interpretaciones y las prácticas de los adultos y excluye cualquier concepción de aquellos como interpretes activos de su mundo. El autor muestra que los niños son por el contrario "razonadores prácticos" de su mundo cotidiano.

Dentro de la misma línea de argumentación Matthew Speier publicó, cuando era miembro de la Universidad de la British Columbia, una serie de estudios sobre la naturaleza de la interacción de los niños con los adultos (Speier, 1970 y 1974:135 -159.) El autor mostró el amplio rango de estrategias dc conversación que era competentemente utilizado por los niños en su interacción cotidiana en actividades tales como saludar, hacer referencias y categorizaciones, jugar, seleccionar términos para dirigirse a otros, preguntar y responder, etc. El observó que los niños eran hábiles usuarios de procesos como obtener permiso para dirigirse a los adultos con pro-secuencias tales como "mami, sabes que?". Esta expresión puede comprender el movimiento previo a una secuencia en la cual el siguiente movimiento lo hace el interlocutor para otorgarle o negarle al niño el permiso para continuar hablando. Esta secuencia puede evidenciar las formas en que los adultos exigen, refuerzan y orientan sus derechos convencionales sobre los niños, de la misma manera que estos se validan para otros grupos considerados como limitados, tal como es el caso de aquellos con retrasos mentales, enfermedades mentales, algunos prisioneros y otros grupos por el estilo. En estos términos, los etnometodólogos y analistas de la conversación perciben la edad adulta y la niñez no como un fenómeno sustancialmente progresivo, sino en términos de una serie de arreglos de convenciones de procedimientos, orientados por los adultos y los cuales determinan los recursos de las 
convenciones de interacción, los cuales a su vez asumen y reproducen las asimetrías entre los adultos y los niños.

Speier va aún más lejos que Mackay al señalar que la concepción de infancia y socialización de los sociólogos convencionales, se fundamenta en su compromiso con la perspectiva adulta y la aceptación de las interacciones adultos-niños. En este sentido, los puntos de vista analíticos de estos sociólogos son ideológicos esta es una de las razones por los cuales Garfinkel sostiene que a pesar de la superioridad que atribuyen los sociólogos convencionales a sus puntos analíticos en relación con el sentido común que informa los análisis de los miembros corrientes de la sociedad, la naturaleza misma del razonamiento sociológico convencional descansa necesaria y constantemente en los puntos de vista del sentido común con recursos tácitos e inexplicados en la construcción de una visión analítica. En contraste, la etnometodología y el análisis de conversación buscan transformar esos recursos del sentido común en los tópicos explícitos del escrutinio analítico.

Más aún, Speier siguiendo las primeras observaciones de Sacks, invocó las observaciones naturalistas de los folcloristas lona y Peter Spie (1959 y 1969) para mostrar que los niños utilizan sus propios recursos culturales compartidos, para interpretar sus interacciones comunicativas con los adultos. Estos recursos se derivan de los dominios culturales de los niños y no de los adultos.

En la comunicación entre adultos y niños -incluidas las charlas en el salón de clase y las situaciones de pruebas educativas puede darse un elemento significativo de contacto cultural (Speier 1976:147).

Desde esta perspectiva, el examen educativo por ejemplo, implica un lenguaje, una cultura y un contexto -saturado de interacción e interpretativamente rico- el cual inter alia involucra una cantidad considerable de arbitrariedades y ad hoc practices por parte del examinador esto sucede tanto en la conducción de la prueba como en la evaluación de los resultados, particularmente cuando el niño da una respuesta que "hace sentido" o es plausible -bien sea desde la perspectiva cultural del niño o de el adulto-, pero que es calificada a pesar de todo, como sustancialmente incorrecta según el patrón evaluativo del examen.

De acuerdo con lo demostrado por los estudios de etnometodología, análisis de conversación y otros trabajos, los resultados de los exámenes son en su quinta esencia producto del trabajo conjunto, aunque corrientemente los examinadores los tratan como productos individuales de los niños que son examinados. Al respecto pueden consultarse Mackay, 1974 y Cicourel et al (1978), cuyo trabajo este campo se deriva en gran medida del trabajo fenomenológico de Schutz. Estudios desde la perspectiva del Análisis de Conversación que reportan diferentes formas de interacción adulto-niño han sido realizados por French, así como por autores tales como Madure (1984) y Wotton (1981 a y 1981 b).

Maynard (1987) realizó también desde esta perspectiva un análisis de las pruebas educativas. Más aún, James L. Heap, utilizando datos de las escuelas primarias canadienses ha permitido una mejor comprensión de la organización social inherente a las actividades de lectura. Más aún, en relación con la evaluación de una destreza determinada, Heap mostró que no es de ninguna manera cierto que los niños utilizan solamente los recursos interpretativos dados por el maestro y la actividad evaluativa misma, así como que tampoco es correcto que los niños comprenden las preguntas del 
examen en términos del marco interpretativo que ha sido presupuesto en el diseño del examen.

En resumen, los estudios de la conversación han cambiado los pilares de los sociólogos. En lugar de usar términos adulto y niño, como recursos esencialmente no examinados para el análisis, han planteado una reorientación que conlleva a tratar esos términos como identificaciones lingüísticas o categorizaciones con usos convencionales, las cuales son captadas y no utilizadas por los analistas, sino por las personas corrientes en la vida cotidiana .. Esta reorientación se mantiene incluso en estudios sobre la cultura infantil y las pruebas educativas. De acuerdo con las observaciones de Spier y otros investigadores un aspecto constante hace referencia a la forma como se define quienes son niños, y quienes son adultos en la situación inherente a las pruebas educacionales. Es el problema de categorización al que se hará referencia en el aparte siguiente.

\section{CATEGORIZACION, NIÑOS Y EDUCACION}

Los primeros analistas de la conversación se preocupaban de manera prioritaria con el fenómeno inherente a la categorización de los miembros de una sociedad -es decir, el aparato intersubjetivo interpretativo compartido por los miembros de una sociedad el cual está lingüísticamente impregnado de agrupaciones para la identificación de las personas, así como de las reglas de procedimiento para el uso de las categorías. Ejemplos ordinarios para la categorización de los miembros de una sociedad incluye definiciones tales como adulto, niño, adolescente, madre, hijo, jugador de tenis, profesor, alumno, etc. Es obvio que cualquier lenguaje posee un gran número de este tipo de categorías convencionales.

Con base en un análisis de cuentos infantiles Sacks observó que estas categorías sirven de referencia para definir los derechos y las obligaciones de la interacción, incluyendo las asimetrías convencionales mencionadas previamente. Al respecto dé las categorías de los miembros comprenden parte del orden lógico y moral, tal como fue señalado por Jayyusi (1984). Sacks observó también la manera como los miembros usan estas categorías de manera tal que se articulan convencionalmente configurando colecciones denominadas "recursos de categorización de miembros" (M.C.D) "Una de estas MCD es "familia" por supuesto, la cual incluye entre otras las categorías de "madre", "padre" e "hijo(a)". De estos patrones pueden derivarse otras colecciones de pares de categorías relacionadas, como por ejemplo padre-hijo y maestro -alumno (Sacks, 1972: 3842). Sacks (1974) formula una regla de consistencia para el uso combinado de categorías, cuando por ejemplo dos categorías son utilizadas de manera cercana en la conversación y cuando pueden devenir del mismo M.C.D..

Algunas actividades y predicados pueden ser también convencionalmente imputados a una categoría dada como por ejemplo "bebé" y "llanto". Atención especial han puesto los analistas de la conversación a los M.C.D que hacen referencia a etapas de la vida., tal como es el caso de Sacks y Atkinson (1980), en los cuales nociones tales como niño y adulto son apenas una parte de un MCD más amplio. Atkinson examina los usos prácticos de la comunicación (por ejemplo, en los juicios de retardo o precocidad), basados en MCD

\footnotetext{
${ }^{*}$ NT: Se hace referencia a las concepciones intersubjetivas que son compartidas, es decir, del conocimiento cultural.

** NT. Esta expresión corresponde en inglés a la frase 'Membership Categorization Device" cuya sigla es la MCD. La traductora utilizará la sigla en inglés para facilitar el acceso a los textos correspondientes en ese idioma, dado que la producción sobre el tema en español es prácticamente inexistente.
} 
correspondientes a etapas de la vida, que incluyen la invocación de derechos y obligaciones. Baker (1984) examinó el uso de estas categorías y aquellas ligadas a las actividades correspondientes a la comunicación adolescente -adulto.

Aunque las categorías de miembros y los recursos de la conversación han sido analizados predominantemente referidos a las personas, se han intentado algunas extensiones a la espera de las referencias geográficas en los cursos de geografía, tal como es el caso de McHoul y Watson (1984).. Otros estudios han hecho extensivo el análisis de las categorías de los miembros a la esfera de los textos escritos, según puede observarse en el estudio de los primeros libros de lectura escolar realizado por Baker y Frebody (1987), En ese trabajo los autores examinan las categorizaciones de miembros según ellas se presentan en un conjunto de prácticas del texto, haciendo énfasis en la manera en la cual se utilizan las categorías de edad y género. En el estudio citado y en otro previo, Baker y Freebody (1986), examinaron las atribuciones convencionales del texto en relación con los predicados mentales y otros de la categoría "niño" y citaron la consideración de Atkinson (1980) para quien "niño" y "adulto" son eventos culturales que los miembros hacen que se sucedan." Otros estudios se han enfocado en el par relacional de categorías "profesor" y "alumno" (o "estudiante"), aunque la con confrontación de estas categorías con las de "adulto" y "niño" no ha sido totalmente abordada. Muchos de los estudios, de la importante colección de Payne y Cuff (1982), sobre el manejo de la interacción en los salones de clase examinan el uso que hacen los interlocutores de las categorías de identificación de miembros (ver el artículo de Payne: 95); así como las relaciones entre el poder y la autoridad (Payne y Hustler, 1981), las cuales son en parte establecidas y reproducidas de manera reconocible por medio del aparato cultural de la categorización de los miembros. Este último es incidentalmente, un estudio que pone en tela de juicio la crítica frecuente que se hace a la etnometodología y al análisis de conversación, en el sentido de que no permiten abordar aspectos pertinentes al poder y a la autoridad.

Este aparte se ha enfocado en las diferentes perspectivas analíticas que han sido aplicadas por los etnometodólogos y los analistas de la conversación en sus estudios de las categorías de miembros de las sociedades, con la esperanza de mostrar que la etnometodología y el análisis de conversación son sobretodo términos para una clase de aproximaciones empíricas que se interrelacionan y no son excluyentes. Dado lo anterior, es posible pasar a discutir la forma como las categorías de miembros son utilizadas por los interlocutores como un esquema de fondo para la distribución de derechos diferenciales de participación en el salón de clase.

\section{DAR LA PALABRA EN EL SALON DE CLASE. Un Análisis Secuencial}

A. W. Mchoul (1978) ha realizado contribuciones significativas para el análisis del sistema de intercambio de turnos en las conversaciones que tienen lugar en el salón de clase. El autor observa que las conversaciones en la clase ocupan una posición intermedia en un arreglo lineal del sistema de intercambio de discursos en el cual el sistema genérico es la conversación ordinaria Los otros sistemas de intercambio de discursos (charlas en el salón de clase, entrevistas de investigación sociológica, careos judiciales, interrogaciones policíacas), son todas derivadas y con algún grado de variación, de transformaciones de la conversación corriente. Ver por ejemplo Sacks, Schegloff y Jefferson (1974). Los sistemas más formales escritos, tal es el caso de las ceremonias litúrgicas de las iglesias, las cuales se acercan al extremo polar de formalidad 
La conversación en el salón de clase aunque no tan estructurada es relativamente formal en el sentido que involucra una tendencia significativa para relacionar ciertas acciones de la conversación, tales como dirigirse a algunas categorías relevantes para cl salón de clase, por ejemplo "maestro", entre mis formal sea el salón de clase es más exigente la preasignación de turnos. Una dimensión central de este sistema de intercambio de conversación corresponde al hecho que los interlocutores se orientan por él y lo activan y manejan de manera rutinaria en el mismo proceso de operarlo.

Para Mc Houl el sistema de convenciones en el salón de Clase excluye el rango de opciones y permutaciones accesibles en la conversación ordinaria. En ésta por ejemplo, quien habla puede seleccionar como interlocutor a cualquier otra persona ue participe en la conversación. En la conversación del salón de clase sin embargo, el estudiante puede seleccionar solamente al maestro como el próximo interlocutor. Cuando el maestro selecciona un interlocutor éste tiene el derecho y la obligación de hablar. Si el maestro no selecciona al siguiente interlocutor a quien está hablando se le permite continuar. Aunque los estudiantes pueden solicitar permiso para hablar, ellos no pueden interrumpir. Los estudiantes pueden escoger solamente entre dos opciones a saber: retornar la palabra al maestro o continuar hablando. Mc Houl elabora cuidadosamente las sutiles complejidades de estas reglas y plantea que solamente el maestro puede dirigir la conversación de una manera creativa.

De acuerdo con MacHoul este sistema de permutación-reducción, sirve para minimizar los sobresaltos, los yacios y la congestión, permitiendo manejar de manera ordenada las dificultades del salón de clase. El autor ratifica estas reglas básicas de conversación en el salón de clase y hace algunas anotaciones sobre como se enmiendan los problemas que surgen en el proceso. Para visión crítica puede consultarse a Heap (1979).

Cuff y Payne en French y Maclure (op. cit) han mostrado como las conversaciones del salón de clase involucran una variedad de formas; en particular, ellos analizaron el contar cuentos en el salón de clase de las escuelas primarias.. Usando los mismos datos Cuff y Hustler publicaron un análisis de conversación significativamente diferente pero complementario. Estos autores señalan que el contar un cuento, cuando quien lo propicia y lo escucha es el maestro, es un recurso de conversación especialmente adecuado para captar una secuencia de cuentos relatados por los alumnos después de que el primer cuento haya sido narrando por el profesor. También puede observarse que el cuento que es propiciado le permite al maestro asumir el papel de eje de distribución de la conversación descrito por Mc Houl en términos de la ubicación de una participación diferencial y el control de los derechos en el salón de clase.

\section{COMENTARIOS FINALES}

Esta breve revisión, por sus mismas características ha dejado de lado muchas de las áreas sustantivas de aplicación de la etnometodología y el análisis de la conversación a la esfera de la educación. Por ejemplo, James L. Heap y su equipo de investigadores en OISE han conducido un trabajo pionero en torno a la enseñanza del uso de computadores en las escuelas primarias canadienses. El profesor Richard Hayman y su grupo de investigación (Dialogue Analysis Research Group) en la Universidad de Calgar también han realizado, entre otros estudios, en el área de educación en computadores y la ¡interacción de grupos minoritarios en el salón de clase. 
En este trabajo, no se ha pretendido de ninguna manera sugerir que la etnometodología y el análisis de conversación son las únicas aproximaciones adecuadas para entender la educación como un fenómeno social. Sin embargo, sí se ha querido indicar que éste abordaje permite captar algunos fenómenos que a pesar de ser centrales han pasado desapercibidos en los análisis convencionales. Esto ha sido posible mediante el uso de una rigurosa metodología sociológica, esencialmente de tipo naturalista.

\section{BIBLIOGRAFIA}

Atkinson, Michael A.

"Some Practical Uses of "A Natural Lifetime",pp. 33-46 in Human Studies, Vol. 3, 1980.

Atkinson, Maxwell, y John Heritage, editores : Structures of Social Action: Studies in Conversation Analysis, Cambridge: Cambridge University Press, 1984.

Baker, C.D.

The "Search for Adultnes": Membership Work in Adolescent Talk', pp. 301-23 en human Studies, Vol. 7, 1984.

Baker, C.D.y p. Freebody

'Representations of Questioning and Answering in Children's First School Books', pp. 45184 en Language in Society, Vol. 15, 1986.

Button, G. y John RE. Lee editores:

Talk and Social Organization ., Clevedon, Avon, England: Multilingual Matters Ltd, 1987.

Cicourel, A.V. et al.

Language Use and School Perfomance, New York: Academlc Press, 1974.

Cuff, E.C. y D.E. Hustler

'Stories and Story Time in an Infant classroom' en French and Maclure (ver adelante), 1981.

Cuff, E.,C. y G.C.F. Payne, editores

:Perspectives in Sociology Second Edition, London: George Allen and Unwin, 1985.

French, P. y M. Maclure,

editores : Adult-Child Conversation London : Crom Helm, 1981.

Garfinkel, H

Studies in Ethnomethodology Cambridge: Polity Press, 1984.

Heap, J.L.

'Classroom Talk: A Critique of McHoult'. Mimeo inédito Department of Sociology In Education, the Ontario Instltute for Studies in Education, Toronto, Ont, 1979.

Heap, J.L. 
What Counts as Reading: Limits to Certainty In Assessment' pp. 265-92 en Curriculum Inquiry, Vol. 10, N0 3, 1980.

Hleap, J.L.

'The Social Organization of Reading Assessment: Reasons for Eclecticism', pp. 39-59 en Payne and Cuff ( ver adelante), 1982.

Heap J.L.

'Ethnomethodology and Education: Possibilities', Journal of Educatíon Thought, 1985a.

Heap, J.L.

Colaboration in Word Processing: The Impact of Technology on Education, The Evolving Role of the Student. Informe final del proyecto Colaboration in Word Processing' to the Ministry of Education, The Ontario. (Mimeo: Department of Sociology in Education, Toronto, Ont., 1985b.

Heritage, j.

Garfinkel and Ethnomethodology, Cambridge: Polity Press, 1984.

Hustler, D. y E.C. Cuff

'Telling a Story Teacher and Pupil Competence in an Infant Classroom' p.p. 14-38 en Payne and Cuff, (ver adelante)., 1982.

Jayyusi, L.

Categorization and the Moral Order, London: Routledge and Kegan Paul, 1984.

Keddie, N.

'Classroom Knowledge', pp. 133-60 en M.F.D. Young, editor:

Knowledge and Control: New Directions for the Sociology of

Education London: Collier - Mc millan, 1971.

Mackay, R.

'Conceptions of Children and Models of Socialization', pp. 180-95 en Roy Turner, editor: Ethnomethodology Harmondswort: Penguin, 1974.

Maynard, D.W.

'The Interactional Substrate of Educational Testing' Paper presented at the conference "Action Analysis and Conversation Analysis', Maison des Sciences de 1' Homme, Paris, France, 30 september, Mimeo, Sociology Department, University of Winsconsin, W1S, USA. ,1987.

Mc Houl, A.W. y D.R. Watson:

"Two Axes for the Analysis of "Commonsense" and "Geographical" Knowledge in Classroom Talk', pp. 281-302 en

The British Journal of the Sociology of Education Vol. 1, 1984.

Mehan, $\mathrm{H}$.

Learning Lessons Cambridge MA.: Harvard University Prees, 1979.

Opie, I. y P. Opie

The Lore and Language of School Children Oxford: The Clarendon Press, 1959.

Opie, I. y P. Opie 
Children 's Games in Street and Playground. London: Oxford University Press, 1969.

Payne G.C.F

'Dealing With a Late -Corner', pp. 90-103 en Payne and Cuff (ver la siguiente cita), 1982.

Payne, G.C.F. y E.C. Cuff, editors:

Doing Teaching : The Practical Management of Classrooms, London: Batsford, 1982.

Payne, G.D.F. y D.E.

Hustler 'Power in the Classroom' S.N.T., 1981.

Sacks, $\mathrm{H}$.

'Sociological description', pp. 1-16 en The Berkeley Journal of Sociology, Vol. 8, 1963.

Sacks H.

'On the Usability of Conversational Data for Doing Sociology', pp. 31-74 en D. Sudnow, editor: Studies in Social Interaction New York: The Free Press., 1972.

Sacks, H.'On the Analysability of Stories By Children', pp.216-32 en Roy Turner, editor: Ethnometho-dology op. cit ., 1974.

Sacks, H. E.A. Schegloff y G. Jefferson : 'A Simplest Systematics for the Organization of Turn-Taking for conversation'., pp. 696-735 en Language ${ }_{1}$ Vol 50.

Speier, M.

How to Observe Face -To-Face Communication: A Sociological Introduction Pacific Palisades, California: the Goodyear Publishing Co., 1974.

\section{Speier, M.}

'The Child as a Conversationalist; Some Culture Contact Features of Conversational Interaction Between Adults and Children', pp. 98-103 en Hammersley, M. and P. Woods, editores: The Process of Schooling, London: Routledge and Kegan Paul., 1976.

Wootton, A.

'The Martagement of Grantings and Rejections by parents in Request Sequences', pp. 5989 en Semiótica Vol 37, 1981a.

Wootton, A.

'Children's Use of Address Terms', pp. 42.58 en French and Maclure (op. cit.)., 1981b. 